\title{
Internal structure of the Jovian simple S-burst obtained with the wavelet analysis technique
}

\author{
G. V. Litvinenko ${ }^{1}$, H. O. Rucker ${ }^{2}$, V. V. Vinogradov ${ }^{1}$, M. Leitner $^{2}$, and V. E. Shaposhnikov ${ }^{3}$ \\ 1 Institute of Radio Astronomy, Krasnoznamennaya St. 4, 61002 Kharkov, Ukraine \\ 2 Space Research Institute, Austrian Academy of Sciences, Schmiedlstrasse 6, 8042 Graz, Austria \\ e-mail: helmut.rucker@oeaw.ac.at \\ ${ }^{3}$ Institute of Applied Physics, Russian Academy of Sciences, Uljanov St. 46, 603600 Nizhny Novgorod, Russia
}

Received 17 February 2003 / Accepted 14 May 2004

\begin{abstract}
The internal structure of the fundamental Jovian decameter radio emission, the simple-shaped millisecond (S-)bursts have been investigated with the continued wavelet transform (CWT) technique. The wide-band data taken for the present analysis have been obtained with the high-frequency and high-time-resolution equipment of a digital spectropolarimeter (DSP) and a waveform receiver installed on the world's largest decameter band radio telescope UTR-2 within the frame of a joint Ukraine-France-Austria-Russia INTAS project. In the wavelet spectra of the investigated events clear signatures of microsecond modulations have been found. These microsecond structures of simple Jovian S-bursts open new perspective on the development of a possible generation mechanism theory, which is briefly discussed.
\end{abstract}

Key words. methods: data analysis - planets and satellites: individual: Jupiter - radio continuum: solar system

\section{Introduction}

Since the detection of the Jupiter decameter emission by Burke \& Franclin (1955) in 1955 great emphasis has been put on the investigation of the sporadic Jovian decameter S-burst radiation (Flagg \& Desch 1980; Genova \& Leblanc 1981; Goertz 1983; Ryabov 1992; Imai et al. 1997; Carr \& Reyes 1999; Litvinenko et al. 1999, 2000; Rucker et al. 2000). However, the generation mechanism of this intriguing phenomenon still remains unclear. Unfortunately, until now there is no general theory for the explanation of whole number of observed parameters (Ryabov \& Gerasimova 1990, and references therein). Thus it is obvious that new observations technologies and theoretical approaches should be applied for the Jovian decameter emission investigations.

The Jupiter decameter (DAM) radiation consists of two types of wide-band noise radio storms, called L-bursts (time scale in seconds) and S-bursts (time scale in milliseconds), with different spectral envelopes and time scales (Ellis 1975). S-bursts manifest themselves as a series of short pulses with durations from a few to tens of milliseconds and are strongly controlled by the Jovian satellite Io, i.e. they are observed only in two particular configurations of the Io-Jupiter-observer angle. Io-related DAM emission monitoring revealed the characteristics of the Jupiter emission zones known as Io-A, Io-B, Io-C, and Io-D and their respective dependencies on Central Meridian Longitude (CML) and Io-phase (Bigg 1964). S-bursts constitute approximately $10 \%$ of the total decameter emission of Jupiter and they produce the most powerful radio emission in the solar system. They are characterized by e.g. spectral form, rapid drifts in the frequency-time plane, "fine" temporal and frequency structure, narrow-band events. The electromagnetic radiation represents a fast extraordinary wave. It is suggested that the emission pattern is a cone with radiation in the cone mantle, and the apex angle that is directed towards the planet. Since the brightness temperature of the radio bursts is substantially higher than the temperature of the Jovian magnetosphere and ionosphere, it is supposed that the generation mechanism is coherent. Following the classification of Carr \& Reyes (1999) the Jovian S-bursts can be divided in two types: a) simple S-bursts appearing in the Fourier millisecondscale dynamic spectrum as a set of sloping thin straight lines with a constant-time half-power bandwidth in the vicinity of $10-20 \mathrm{kHz}$; b) complex S-bursts with the instantaneous bandwidths much larger than those of simple S-bursts.

The main goal of the present work is to investigate the peculiarities of the internal structure of simple S-burst internal structure. It should be noted that a first investigation of the Jovian simple S-burst sub-structure was made by Carr \& Reyes (1999) and further developed by Carr (2001). They analyzed Jovian S-burst signals recorded at $26.3 \mathrm{MHz}$ with the highly sensitive 640 dipoles antenna array at the University of Florida Radio Observatory. The processed data were presented in the form of receiver base-band output voltage data as a function of time. 
Their most important results concerning the sub-millisecond structure of simple S-bursts are the following: a) the discovery of subpulses, which are successive deep envelope modulated subpulses that are always clearly observable with high time resolution (better than $30 \mu \mathrm{s}$ ) in the plot of power density versus time; b) the discovery of intervals of phase coherence that begin and end abruptly, and occur mainly during the more intensive subpulses. So the generation process has to be strongly phasecoherent. In the paper by Carr (2001) two interesting empirical models of the obtained S-burst microstructure were proposed. First, the so-called "narrow-band fluctuation model" assumes that the burst waveform represents the statistical fluctuations of the sum of the instantaneous amplitudes of a large number of closely spaced but randomly phased frequency components. Second, the "pulse superposition model" is based on the assumption that the S-burst signal represents the superposition of a group of pulses from closely spaced but independent shortlived emission centers which are nearly monochromatic but slightly different in frequency. Both models need to be considered in details and connected to the generation processes at the source, a task still to be done. Further to the work of Carr (2001) a nonlinear feedback mechanism (Helliwell 1967; Melrose 1986) for the generation of Jovian S-burst was reexamined by Willes (2002) from the point of view of recent observations of S-burst phase coherence. In the feedback model an interaction between waves and particles of loss-cone anisotropy is assumed to take place in the "interaction region" (IR), which is drifting along the field lines. Particles entering the drifting IR are phase-bunched by a wave which is already emitted at cyclotron frequency and leaving the IR, and later as these particles approach the other side of the IR they generate a new wave at a different frequency. It was shown by Willes (2002) that the same mechanism, operating in two separate regimes, can produce both constant-frequency (zero frequency drift) and rapid-frequency-drift emissions.

In the present work the data of the simple S-bursts obtained with the high-spectral-resolution equipment of a digital spectropolarimeter (DSP) and the broadband waveform receiver (WFR) installed on the decameter band radio telescope UTR-2 at Kharkov (Ukraine) have been analyzed with the wavelet analysis method.

The paper is structured as follows: after a short description of the observational equipment and data, some information is given on the wavelet transform analysis. Finally, the new results are presented and discussed in detail.

\section{Observations}

Previous observations of DAM emission were based on the use of broadband multi-channel systems with efficient data processing techniques for interference elimination as well as selection and integration of non-polluted frequency channels. This processing was then followed by an algorithm performing burst detection and parameter determination. However, this system, first, could not provide sufficient temporal and frequency resolution, and second, the obtained data presented only the amplitude (intensity) variations versus time in separate frequency channels. At present, the high technology of the
Digital Spectropolarimeter (DSP, Kleewein 1997) and of the recently developed Waveform receiver (WFR, Leitner 2001) with very high resolution in frequency and time, provides the possibility to obtain complete information about the radio signal over the broad-band frequency range, and thus opens new perspectives for the investigators.

The Ukrainian T-shaped $(1600 \times 60 \mathrm{~m}$ size of telescope $)$ radio telescope array (Braude et al. 1978) is one of the most effective and sensitive instruments for investigations of astronomical radio sources in the decametric range. Within the frame of the Ukraine - Austria - France - Russia INTAS project ("New Frontiers in Decameter Radio Astronomy") observations of the Jovian decameter emission have been performed with UTR-2 (Konovalenko et al. 2001; Rucker et al. 2001). During the observation campaign $\mathrm{C} 2$ the telescope was equipped with the broad-band waveform receiver (WFR) (Leitner 2001; Leitner \& Rucker 2001) which runs in combination with the digital spectropolarimeter (DSP) (Kleewein 1997; Kleewein et al. 1997). The DSP provides the ultimate spectral analysis capability of performing real time digital signal processing. The signal is digitized immediately after the HF-preprocessing stage. This type of digital spectropolarimeter can operate down to a time resolution of one millisecond with 1024 frequency channels over an instantaneous bandwidth of $12.5 \mathrm{MHz}$. The measured linear dynamic range for broadband noise is greater than $60 \mathrm{~dB}$.

The waveform receiving system (WFR) presents a fully digital broadband recorder. The WFR reaches the maximum theoretically possible time-frequency resolution, where the product of frequency and time is approximately 1 . The dynamical range of the waveform receiver is about $70 \mathrm{~dB}$, the bandwidth in the single channel mode is $25 \mathrm{MHz}$. Due to the enormous amount of data produced within one second, the limited data storage enables a continuous acquisition duration of only $6 \mathrm{~s}$. The main concept of the waveform receiver is to store the original signal waveform. The sampling rate of the WFR is set to $50 \mathrm{MHz}$. The digitized data are transferred via the PCI-bus to the internal memory ( $\sim 40$ megabyte) of a standard personal computer system.

\section{Wavelet analysis method}

Signal processing of the Jovian DAM emission is usually based on the Fourier analysis which gives important but limited information about the spectrum of the signal, but is not wholly appropriate for treating non-stationary processes like the sporadic DAM radiation. The main drawback of the Fourier method is the impossibility to obtain the simultaneous localization of a signal in the time and frequency domain because of the "uncertainty relation". Recently, several effective mathematical approaches, such as the wavelet transform, the Wigner-Ville technique and so on, are actively used for the investigations of non-stationary and non-linear processes.

During the last decade the wavelet analysis method has been successfully applied in many areas of signal processing (Mallat \& Hwang 1992; Wilson et al. 1992). The wavelet transform is a mathematical technique that makes it possible to divide data, function or operators into different frequency components, and then considers each component with a 
resolution matched to its scale. The wavelet transform yields a time-frequency description similar to the windowed Fourier transform, but with an important difference which consists in the shapes of the analyzing functions (Daubechies 1988). In the Fourier transform all the used window functions are complex exponential functions with the same envelope "filled in" with higher frequency oscillations. For a one-dimensional signal $s(t)$ the windowed Fourier transform is the following

$\hat{F}(\omega, t)=\int_{-\infty}^{+\infty} s\left(t^{\prime}\right) g\left(t^{\prime}-t\right) \exp \left(-l \omega t^{\prime}\right) \mathrm{d} t^{\prime}$,

where $g(t)$ is the windowing function.

So, $\hat{F}(\omega, t)$ is the signal decomposition over the family of functions $g\left(t^{\prime}-t\right) \exp \left(-l \omega t^{\prime}\right)$ (base functions) produced from one function by the transfers in time and transfers in frequency. All the window functions, regardless of the frequency value, have the same width. A choice of $g(t)$ with large width makes it possible to analyze the low-frequency components but with the loss of short high-frequency phenomena if the width of $g(t)$ is larger than the distance between these phenomena. In contrast, small $g(t)$ does not make it possible to allow to analyze the lowfrequency particulars of signal because of the harmonic content of $g\left(t^{\prime}-t\right) \exp \left(-l \omega t^{\prime}\right)$ which has to include at least one period. So the time resolution is defined by the width of the windowing function. All this means that in the windowed Fourier transform it is impossible to get the optimal time-frequency localization for analyzing of the whole signal.

Contrary to this, the wavelets have time-widths adapted to their frequency: wavelets of high frequency are very narrow, whereas wavelets of low frequency are much broader.

For a one-dimensional signal $s(t)$ the wavelet transformation consists in their decomposition over the family of functions $\psi_{a, t^{\prime}}=\frac{1}{\sqrt{a}} \Psi\left(\frac{t^{\prime}-t}{a}\right)$ of functional Hilbert space $L_{2}(R)$ produced from the mother-wavelet $\Psi\left(t^{\prime}\right)$ (a soliton-similar function) by scale transformations and the shifts (the scale parameter is $a \sim 1 / \omega$, the shift parameter is $t$ ). The wavelet transform exists in two main types: the continuous wavelet transform (CWT) and the discrete wavelet transform (DWT). In the CWT the dilation and translation parameters $a \in R_{\oplus}$ and $t \in R$ vary continuously ( $R$ is set of real number, $R_{\oplus}$ is set of positive real numbers). In the DWT both parameters take only discrete values $a_{m}=1 / 2^{m}$ and $t_{n}=n / 2^{m}$.

The wavelet transform is able to "zoom in" on very shortlived high frequency phenomena, such local periodicity or nonstationarities in the signals.

The continuous wavelet transform (which in general is a complex function) for one-dimensional signal $s(t)$ represents as

$W(a, t)=\int_{-\infty}^{+\infty} s\left(t^{\prime}\right) \psi_{a, t}\left(t^{\prime}\right) \mathrm{d} t^{\prime}$,

where $\psi_{a, t}(t)=\frac{1}{\sqrt{a}} \Psi\left(\frac{t^{\prime}-t}{a}\right)$ is the family of wavelet functions (base functions) which are constructed from the "motherwavelet" function $\Psi(t)$ by transformations and shifts. So the wavelet transform performs a very simple time-frequency transformation of one dimensional signal $s(t)$ into dimensional wavelet image $W(a, t)$ defined on upper half plane $\left[R_{\oplus} \times R\right]$.
The main characteristic of the wavelet functions is its time frequency localization due to the high localization of the "mother-wavelet" (soliton-similar function), i.e. wavelet functions $\psi_{a, t}(t)\left(\equiv \psi_{n}^{m}\right)=0$ outside a short limited interval. This interval is dependent on the scale $a$. Through the uncertainty condition the time resolution is also dependent on the scale. Wavelets of high frequency (small scales) are very narrow, whereas wavelets of low frequency (large scales) are much broader.

The mother-wavelet function $\Psi\left(t^{\prime}\right)$ (analyzing-wavelet) should moreover satisfy the admissibility condition

$\int_{0}^{+\infty} \frac{1}{\omega} \hat{\psi}(\omega) \mathrm{d} \omega=\int_{-\infty}^{0} \frac{1}{|\omega|} \hat{\psi}(-\omega) \mathrm{d} \omega=C_{\psi}<\infty$,

where $C_{\psi}$ is a const, and $\hat{\psi}$ represents the Fourier transform of $\Psi\left(t^{\prime}\right)$. The finiteness of $C_{\psi}$ restricts the class of functions, which can be used as mother-wavelet. Usually the normalization of mother-wavelet has been chosen so that $C_{\psi}=1$. If the condition (3) is satisfied the wavelet transform is inverted and an initial signal $s(t)$ can be recovered from its wavelet-image by the following:

$s(t)=\int_{0}^{+\infty} \int_{-\infty}^{+\infty} W\left(a, t^{\prime}\right) \Psi_{a, t^{\prime}}(t) \frac{\mathrm{d} a \mathrm{~d} t^{\prime}}{a^{2}}$.

Wavelet transform is linear and able to conserve the energy:

$\int_{-\infty}^{+\infty}|s(t)|^{2} \mathrm{~d} t=\int_{0}^{+\infty} \frac{\mathrm{d} a}{a^{2}} \int_{-\infty}^{+\infty}|W(a, t)|^{2} \mathrm{~d} t$

For the purpose of our work we use the Morlet-wavelet which is satisfied (3).

One major difference between most applications of wavelets is the choice of the mother wavelet $\Psi(t)$. For a correct choice of $\Psi(t)$ one needs to know something about the characteristics of the signal to be analyzed and the features of the problem to be solved. Our investigation showed in the case of the Jovian S-bursts that for forming the orthogonal basis the complex Morlet wavelet with a limited definition field seems to be the best as the basis mother wavelet function. Morlet wavelet is defind as (Grossman et al. 1989)

$\Psi_{M}(t)=\mathrm{e}^{-t^{2} / 2} \mathrm{e}^{t \omega_{0} t}$

where $\omega_{0}$ is the central frequency of the wavelet. This mother wavelet function has a high resolution in the frequency as well as in the time domain.

\section{Signal preprocessing}

Before starting to analyze the "superfine" structure of the simple S-burst with the wavelet transformation method the data have to be prepared. The main aim of the data preprocessing is the individualization of one simple S-burst and further signal translation to the lower frequency region. First, the signal has to be filtered to attenuate all frequency bands except the S-burst bandwidth, and to distinguish any interference. For this procedure the recursive Butterworth band filter of 15th order was used (Smith 1999). This filter makes it possible ro reduce the harmonics contamination in $30 \mathrm{~dB}$ in a range of $1 \mathrm{MHz}$ over 


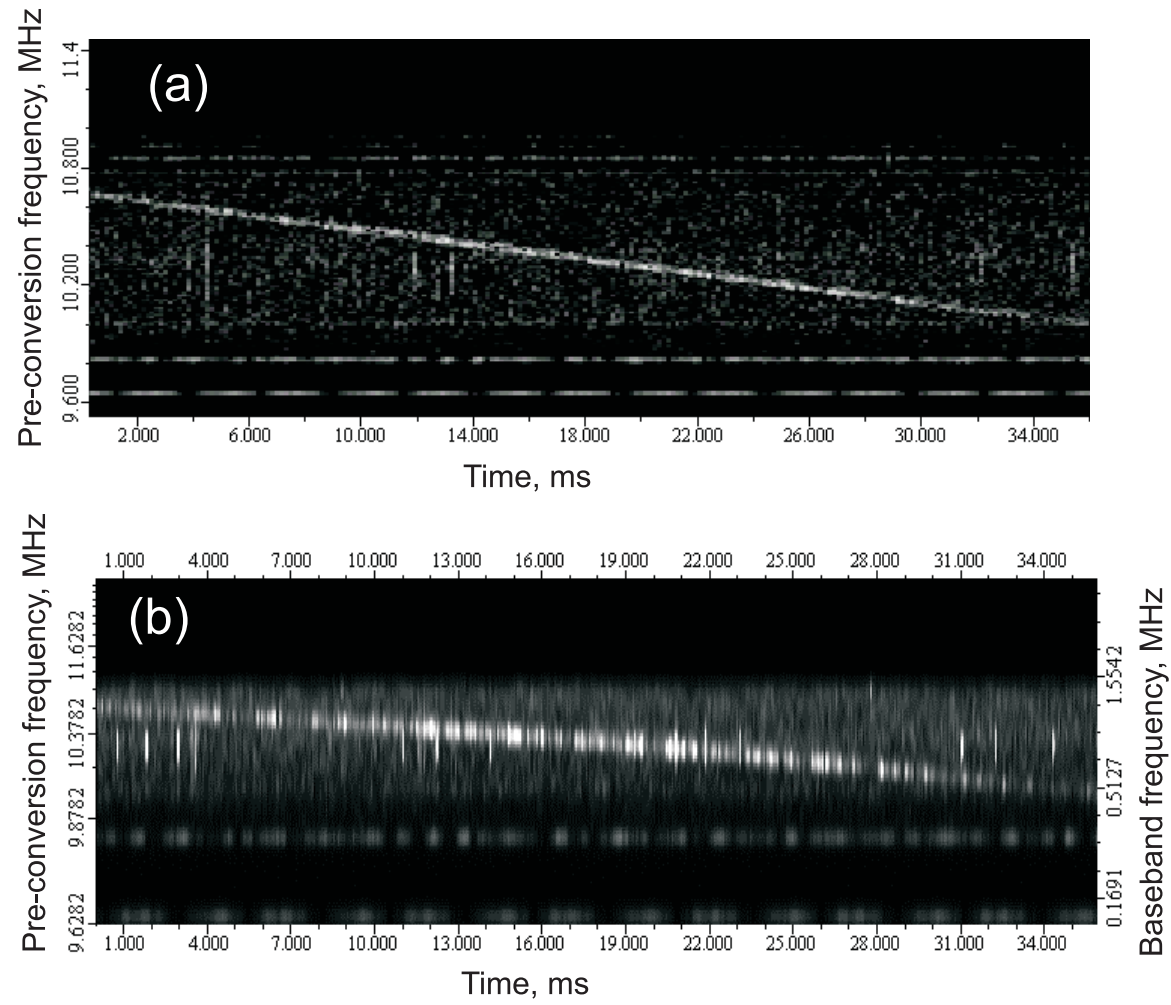

Fig. 1. a) Fourier spectrum of the single S-burst (Io-C event, February 26, 2000). b) Wawelet spectrum of the same burst. the pass-band. Second, the signal has to be multiplied with the harmonic signal of the lower boundary frequency of S-burst band. The multiplication gives a residual and while summing spectral components in the resulting signal. Then only the lowfrequency band is used. The reason for this down-converting to the lower frequencies is, first, to reach the domain of applicability of the continuous wavelet analysis for narrow-band signals, and, second, to reduce the noise in the subsequently obtained wavelet spectrum.

\section{The results obtained by the wavelet transform method}

In Fig. 1 a characteristic example of processed S-burst emission data is shown. In the top panel (a) the simple linear-drift S-burst (a segment of about $35 \mathrm{~ms}$, with an initial data sampling rate of $50 \mathrm{MHz}$ ) dynamic spectrum (Fourier spectrogram) is presented. The horizontal dashed lines in the picture represent radiation of narrow-band fixed frequency radio stations, and vertical lines represent wide-band pulse influences. This signal was recorded during an Io-C storm on February 26, 2000, by the waveform receiver connected with the digital spectropolarimeter installed on the UTR-2 telescope at Kharkov. This spectrum shows that the burst looks like a continuous chirp signal with rather constant characteristics through the time-frequency plane. The signal duration at a fixed frequency is approximately $1 \mathrm{~ms}$. During pre-processing the signal is down-converted to lower frequencies (the frequency shift is 9.5 MHz, see right axis of Fig. 1b). The total frequency bandwidth of a burst is approximately $1 \mathrm{MHz}$. The burst drift rate is $-27 \mathrm{MHz} / \mathrm{s}$, and it was determined with high accuracy from calculating the frequencies with the maximum power spectral density along the burst track. The distribution of the maximum power density with time shows that the more intensive part is located in the center of the spectrum. A similar phenomenon was also noted in the work of Carr \& Reyes (1999).

In the bottom panel of Fig. 1b the wavelet spectrum of the same event (Fig. 1a) is given. This picture was obtained with a sample rate of $14.3 \mathrm{kHz}$ due to the averaging and resampling applied to the initial data set (initial sample rate $50 \mathrm{MHz}$ divided by 3500). One can see that the wavelet spectrum, unlike the Fourier spectrum, shows a very intriguing time structure of the simple S-burst signal.

It need to be noted that the receiver baseband output is an alternating voltage which after was used as initial data for the subsequent necessary calculations as Fourier spectrum and wavelet spectrum. This voltage is digitized at a sampling rate that is many times higher than the dominant frequency of the voltage oscillation. In the subsequent computer processing each digitized voltage value is squared, converting it to power (assuming output impedance to be $1 \mathrm{Ohm}$ ). This quantity is referred to as the total power, $P(t)$ and over the Fourier transform can be expressed as:

$P(t)=\int_{-\infty}^{+\infty}|\hat{F}(\omega, t)|^{2} \mathrm{~d} \omega$

and by wavelet transform as:

$P(t)=\int_{-\infty}^{+\infty} q(a, t) \mathrm{d} a$,

where $q(a, t)=|W(a, t)|^{2} / a^{2}$ and $a \sim 1 / \omega$.

Figure $2 \mathrm{a}$ shows a wavelet spectrum segment from Fig. $1 \mathrm{~b}$ (time interval from 18 through $23 \mathrm{~ms}$ ) with $\sim 7$ times higher time resolution and Fig. $2 \mathrm{~b}$ shows the time profile of wavelet 

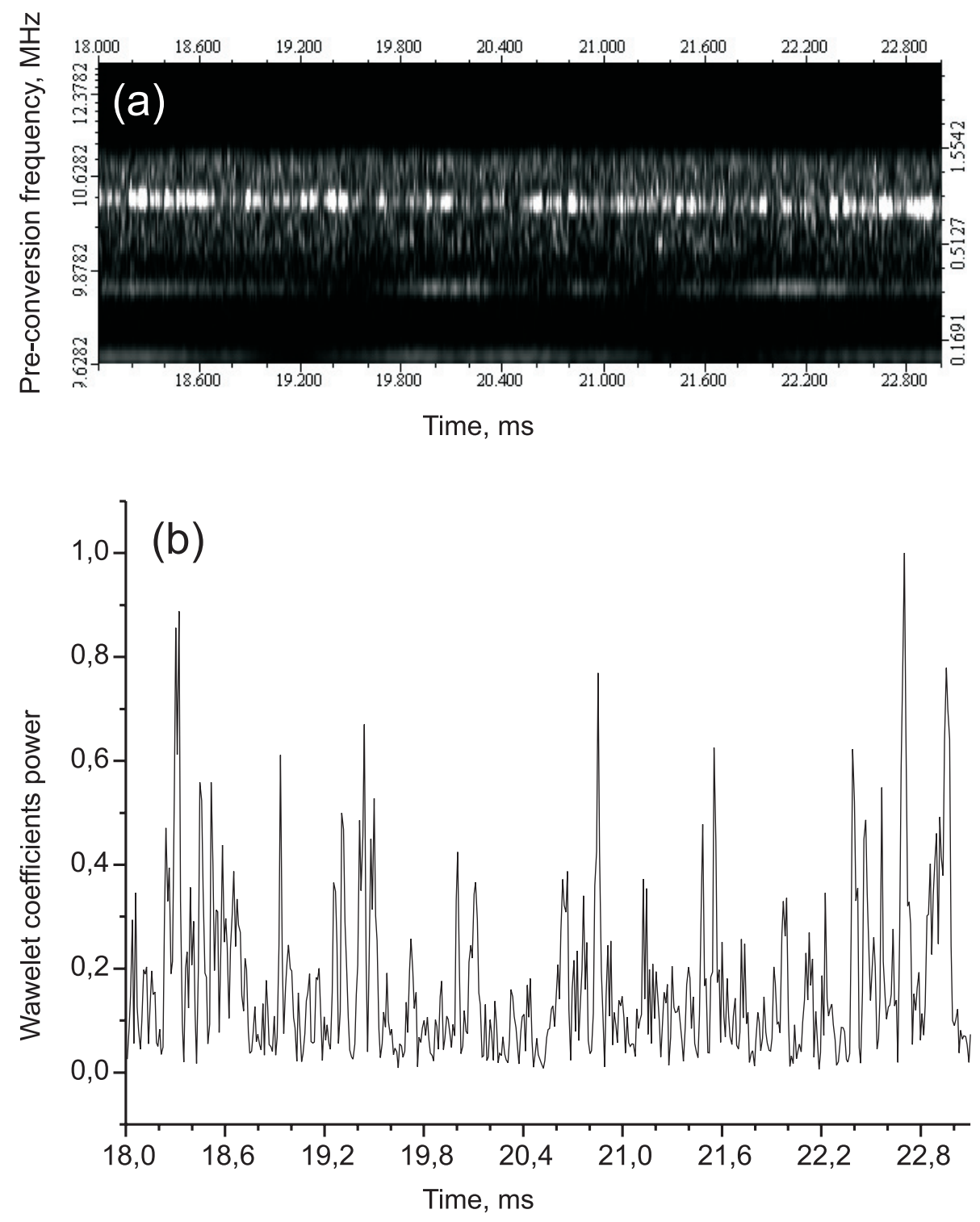

Fig. 2. a) Part of the wavelet spectrum from Fig. 1b obtained with higher time resolution. b) Corresponding time profile of the wavelet coefficient power.

coefficients corresponding to the maximum power along the S-burst track. Obviously the simple S-burst is made up of a series of clear separated pulses with a time duration in the order of microseconds. Aiming towards a more detailed picture of the burst substructure, in the Fig. 3a we choose some intensive interval (18.2 to $18.8 \mathrm{~ms}$ ) and enlarged to the highest possible time-frequency resolution (sample rate $50 \mathrm{MHz}$, number of time points 30000 , time length of complex Morlet mother-wavelet used is $3 \mu \mathrm{s}$ ). As can clearly seen from this picture the internal structure of the simple S-burst signal consists of very short pulses (duration 6-15 $\mu \mathrm{s}$ ) which are, in turn, grouped. In the work of Carr \& Reyes (1999) these groups named "subpulses". It was shown in Fig. 4 that these groups or "subpulses", as obtained by the method of Carr \& Reyes (1999), and those derived by wavelet techniques exhibit a clear correspondence: the signal power and the wavelet coefficients' power have a high degree of cross-correlation. It needs to be strongly stated that this is the first independent verification of the existence of the subpulses reported by Carr \& Reyes (1999).
It should also be noted that in the segment of S-burst event considered a period of grouping of $\approx 0.1 \mathrm{~ms}$ can be determined (see, e.g., Fig. 3a). However, the nature of this quasi-periodic phenomenon needs further investigation. This rather interesting result, concerning not only the phenomenon of "subpulses", but also their "superfine" internal structure, may be due to both the use of advanced observational technology and to the application of the wavelet transform method. Simple analysis of the obtained "superfine" burst structure shows the following average parameters:

- instantaneous frequency band of one separated microsecond pulse: $100-300 \mathrm{kHz}$;

- duration of one separated microsecond pulse: $6-15 \mu \mathrm{s}$;

- time interval between consecutive microsecond pulses: $5-25 \mu \mathrm{s}$.

Thus the instantaneous frequency band of emission is of the order of $200 \mathrm{kHz}$, and the excitation increment of instability, 

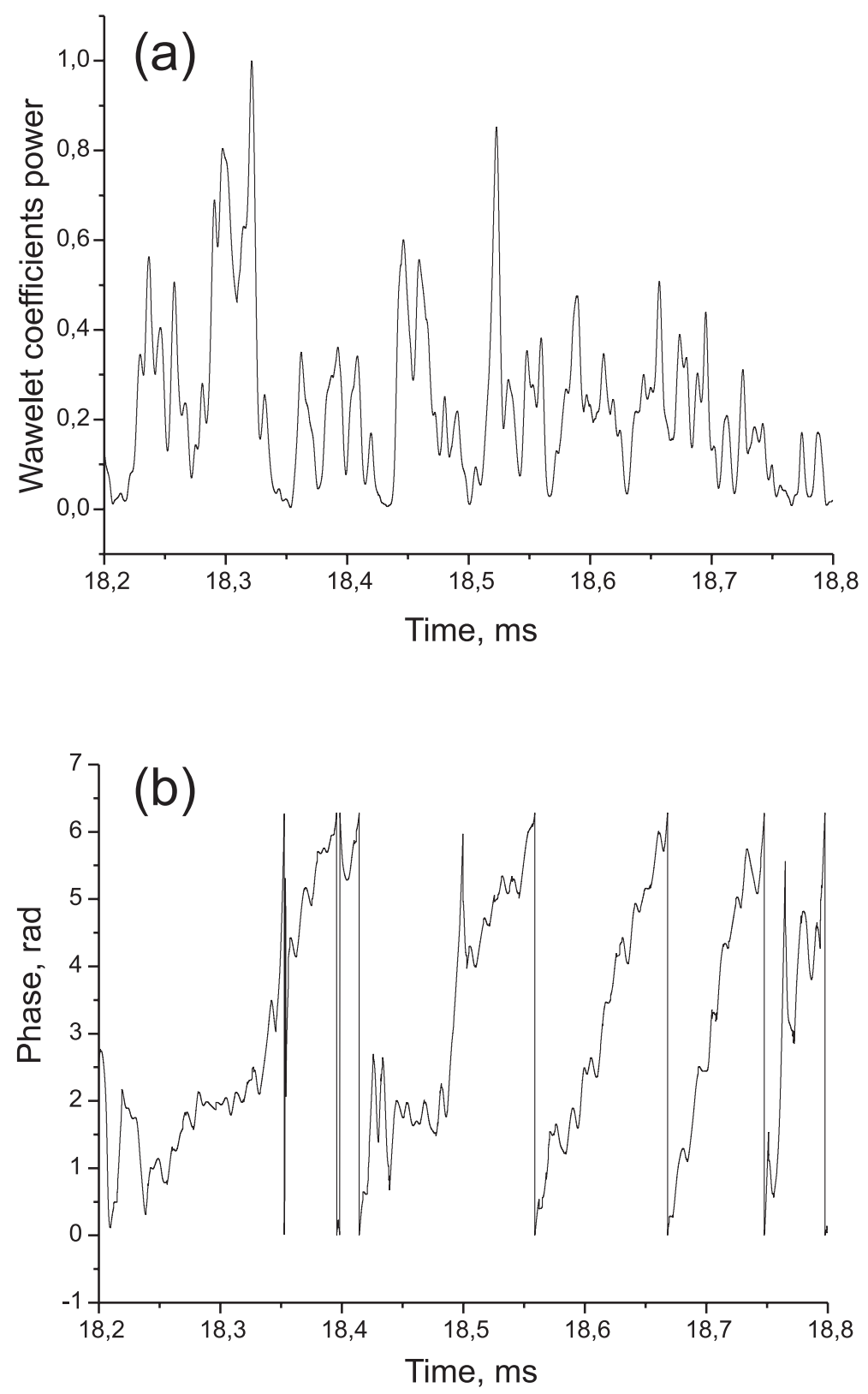

Fig. 3. a) Amplitudes of wavelet coefficients versus time for the considered intensive S-burst segment. b) Corresponding phase variation with the time.

which is supposed to be the cause of the decameter S-burst generation, has a narrow frequency band $\Delta f / f \simeq 10^{-2}$.

It was also found that one separate pulse occupies a specific frequency interval, which we mentioned above as instantaneous frequency band, but has practically no clearly defined frequency drift with time (see, e.g., Fig. 2a). It seems that the frequency drift of the simple burst as a whole results from frequencies decreasing with time for each subsequent pulse. In some parts of the analyzed S-burst the microsecond structure has a quasi-periodical character with a modulation period in the range of from 10 to $50 \mu \mathrm{s}$. However, it is not possible to draw any conclusions about the origin of this quasi-periodic phenomenon.

On the basis of a visual review of a large number of simple S-burst wavelet spectra it can be noted (see Fig. 1b) that the signal intensity is higher in the central part (versus time) of the respective burst than at the edges in the time domain.

To check the result of Carr \& Reyes (1999) concerning the simple S-burst phase behaviour within the subpulses, the following procedure has adopted (see, e.g. Fig. 3 where the limited time segment 18.2 to $18.8 \mathrm{~ms}$ was considered). The relative signal phase variation with time has been retrieved from the variation of the corresponding wavelet coefficient phases. The signal phase variation obtained was then compared to the phase of the hypothetical sinusoidal signal that is the best match over the time segment considered. For this event at least two intervals of approximately constant phase were found (see Fig. 3b): $18.27-18.34 \mathrm{~ms} ; 18.44-18.49 \mathrm{~ms}$ at frequency $0.862 \mathrm{MHz}$ (corresponding to $10.362 \mathrm{MHz}$ before down shifting). The vertical lines in Fig. $3 b$ correspond to the $2 \pi$ phase jump. From a comparison between Figs. $3 \mathrm{a}$ and $3 \mathrm{~b}$ it can be seen that intervals 

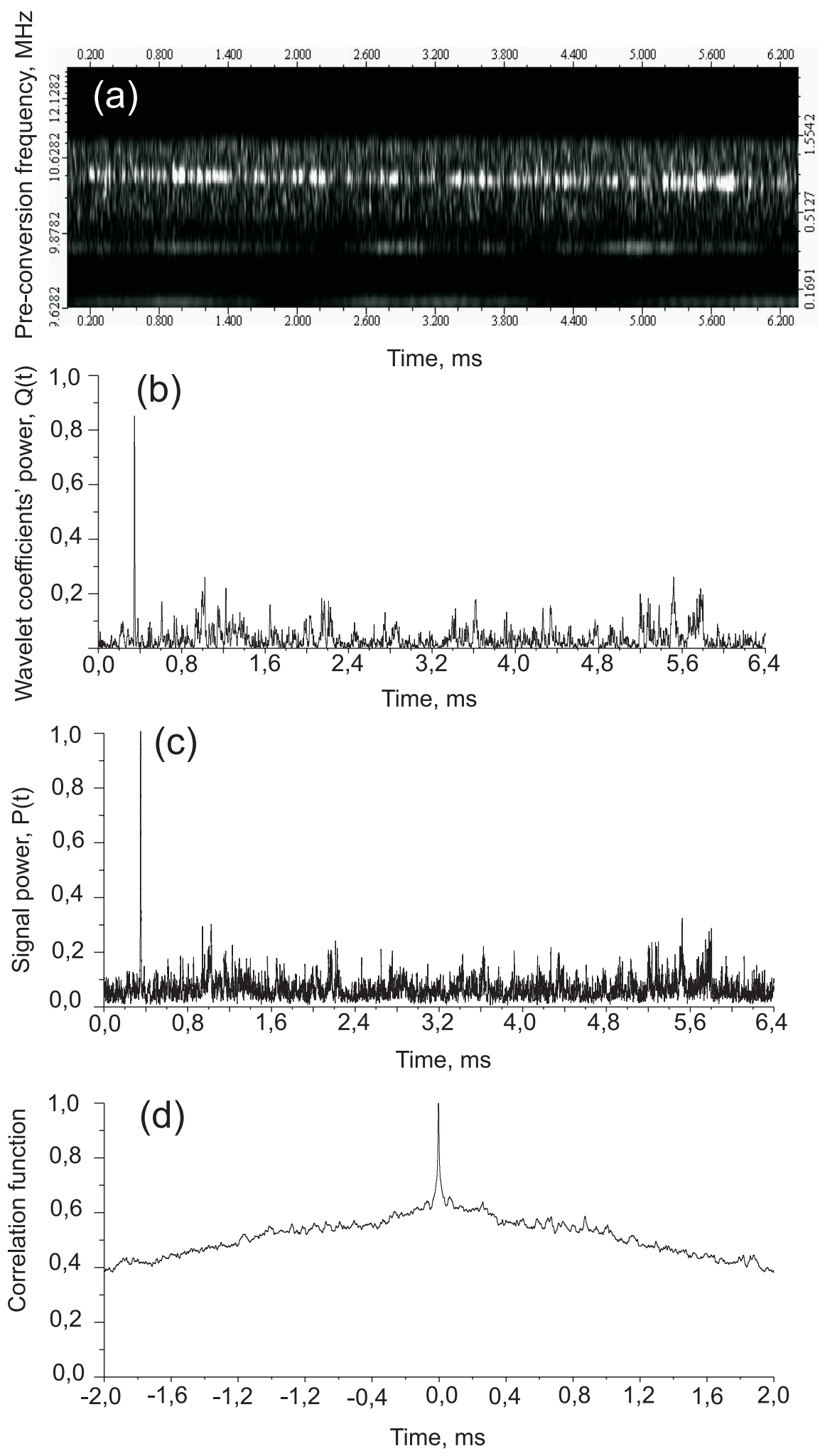

Fig. 4. a) Wavelet imaging of S-burst segment (time interval 14-20.4 ms from Fig. 1a. b) Wavelet power coefficients of the given segment along the burst. c) Signal power obtained from the initial data for the corresponding segment. d) Cross-correlation function between the wavelet power coefficients b) and signal power $\mathbf{c})$.

of constant phase correlate exactly with the intensive groups of microsecond pulses. So the microsecond pulses within the group are phase coherent. This supports the result reported by Carr \& Reyes (1999), Carr (2001). Application of the wavelet analysis technique made it possible not only to determine the subpulses that exist within a simple S-burst but also to show their inherent microsecond pulse structure.

\section{Possible generation models}

The microsecond structure of a simple Jovian decameter S-burst raises the question about the possible mechanism able to form this structure. Several facts must be considered. First, a mechanism that generates S-bursts itself create microsecond modulation processes. Second, the initial emission 
characteristics can be changed along the propagation path from the source region to the observer. According to our present knowledge about the scales of the processes in the Jovian magnetosphere, the interplanetary medium, the Earth magnetosphere and ionosphere, these time scales can certainly not be compared to those microsecond structures.

It is now generally accepted that the Jovian radiation consists of fast extraordinary waves. Since the brightness temperature of the radio bursts is substantially higher than that of the ionosphere and magnetosphere, the Jovian decameter radio emission mechanism should be coherent. In this context the S-bursts are usually connected with the instabilities of electromagnetic and plasma waves near the upper-hybrid frequency which is caused by groups of hot electrons with different anisotropic distribution functions. When the generation mechanism of an extraordinary wave is studied, two possible processes should be considered: 1) the direct excitation of such a wave by non-equilibrium particles in the source (cyclotronmaser mechanism); 2) the generation of a plasma wave near the upper-hybrid frequency with a subsequent transformation into a fast extraordinary wave (plasma mechanism). On the basis of these ideas a number of generation models have been proposed.

Based on the process of cyclotron-maser emission of an unstable electron population, which can to some extent explain the modulation in the Jovian S-burst internal structure, the most promissing generation model seems to be feedback model (Helliwell 1967; Melrose 1986; Calvert et al. 1988; Willes 2002). However, despite the fact that the last Willes modifications of the feedback model makes it possible to obtain an interesting theoretical interpretation of observing sub-structure of S-burst, this conclusion needs to be verified by further detailed calculations.

A plasma model proposed by Zaitsev et al. (1986) for the Io phase dependent S-bursts in Jupiter decameter radio emission may explain the obtained results. In this work the source region was considered to be a multi-component system composed of equilibrium plasma and an admixture of losscone ions and electrons. The loss-cone electrons excite plasma waves near the upper-hybrid frequency. The plasma waves convert into an extraordinary electromagnetic wave due to induced scattering by a beam of superthermal ions or electrons. Under specified conditions a pulsation regime of the plasma wave conversion into electromagnetic waves leads to the internal microsecond character of the simple S-bursts.

\section{Conclusion}

In the present work the internal structure of the simple Jovian decameter S-burst was analyzed on the basis of conceptually new observational technology and the application of the wavelet transform technique. The presence of microsecond pulse groups within the emission (Carr \& Reyes 1999) has been investigated. We have made a comparison between the results obtained by the wavelet method and those yielded by the methodology of Carr \& Reyes (1999). Both techniques apparently lead to an identical conclusion, namely that there exist subpulses during periods of time of phase coherence. In our opinion, the wavelet technique has several advantages, like the possibility to reach very high time resolution (e.g. the definition of the "superfine" structure of "subpulses"), and the more straightforward numerical procedure for getting similar results.

The detection of the internal microseconds structure of simple Jovian S-bursts opened new perspectives, in particular, for developing a theory of possible generation mechanism. This needs, of course, further work, statistical examinations on a broad basis and, as a further step, the consideration of the complex S-burst events. The complex S-burst structure may involve propagation and superposition effects in addition to sophisticated radio burst generation processes.

Acknowledgements. This work has been supported by the Commission for International Cooperation of the Austrian Academy of Sciences. The authors would like to thank Prof. V. V. Zaitsev and Prof. A. A. Konovalenko for fruitful discussions and help, the referee Prof. T. D. Carr for his notes and comments.

\section{References}

Bigg, E. K. 1964, Nature, 203, 1008

Braude, S. Ya., Megn, A. V., Ryabov, B. P., et al. 1978, ASS, 1, 3

Burke, B. F., \& Franklin, L. L. 1955, JGR, 60, 213

Calvert, V. V., Leblanc, Y., \& Ellis, G. R. A. 1988, ApJ, 976

Carr, T. D. 2001, New Clues from the Microstructure of Jupiter's S-Bursts, in Planetary Radio Emissions V, ed. H. O. Rucker, M. L. Kaiser, \& Y. Leblanc (Vienna: Austrian Academy of Science Press), 77

Carr, T. D., \& Reyes, M. D. 1999, JGR, 104, 127

Daubechies, I. 1988, Commun. Pure Appl. Math., 41, 909

Ellis, G. R. A. 1975, Nature, 253, 415

Flagg, R. S., \& Desh, M. D. 1980, JGR, 84, 4238

Genova, F., \& Leblanc, Y. 1981, A\&A, 98, 133

Goertz, C. K. 1983, JGR, 81, 3368

Grossman, A., Kronland-Martinet, R., \& Morlet J. 1989, Reading and understanding continuous wavelet transforms, in Wavelet, Time-Frequency Methods and Phase Space, ed. J. M. Combes, A. Grossman, \& Ph. Tchamitchian (New York: Springer-Verlag), 2

Helliwell, R. A. 1967, JGR, 72, 4773

Imai, K., Wang, L., \& Carr, T. D. 1997, JGR, 102, 7127

Kleewein, P. 1997, Doctoral Thesis, University of Graz

Kleewein, P., Rosolen, C., \& Lecacheux, A. 1997, New Digital Spectrometers for Ground Based Decameter Radio Astronomy, in Planetary Radio Emissions IV, ed. H. O. Rucker, S. J. Bauer, \& A. Lecacheux (Vienna: Austrian Academy of Science Press), 349

Konovalenko, A. A., Lecacheux, A., Rosolen, C., \& Rucker, H. O. 2001, New Instrumentation and Methods for the Low Frequency Planetary Radio Astronomy, in Planetary Radio Emissions V, ed. H. O. Rucker, M. L. Kaiser, \& Y. Leblanc (Vienna: Austrian Academy of Science Press), 63

Leitner, M. 2001, Doctoral Thesis, University of Graz

Leitner, M., \& Rucker, H. O. 2001, Waveform Analysis Techniques of Jovian S-Burst Observations, in Planetary Radio Emissions V, ed. H. O. Rucker, M. L. Kaiser, \& Y. Leblanc (Vienna: Austrian Academy of Science Press), 91 
Litvinenko, G. V., Rucker, H. O., Vinogradov, V. V., et al. 1999, Preprint No. 114, Space Research Institute

Litvinenko, G. V., Rucker, H. O., Vinogradov, V. V., et al. 2000, Radio Sci., 35, 1445

Mallat, S., \& Hwang, W. L. 1992, IEEE Trans. Inform. Theory, 38, 617

Melrose, D. B. 1986, JGR, 91, 7970

Rucker, H. O., Boudjada, M. Y., Lecacheux, A., et al. 2000, Observations and Analysis of Jupiter Millisecond Radiobursts, in Am. Astron. Soc., DPS Meet., 25

Rucker, H. O., Lecacheux, A., Konavalenko, A., \& Leitner, M. 2001, New frontiers in decameter radio astronomy, in Planetary Radio Emission V, ed. H. O. Rucker, M. L. Kaiser, \& Y. Leblance (Vienna: Austrian Academy of Science Press), 51
Ryabov, B. P. 1992, Jovian S-Emission: Decametric High Sensitivity Observations and Model of Radiation Source, in Planetary Radio Emissions III, ed. H. O. Rucker, S. J. Bauer, \& M. L. Kaiser (Vienna: Austrian Academy of Science Press), 125

Ryabov, B. P., \& Gerasimova, N. N. 1990, Sporadic radio emission of Jupiter at decameter wavelength (Kiev: Naukova Dumka)

Smith, S. W. 1999, The scientist and engineer's guide to Digital Signal Processing, Second edition (San Diego, California: California Technical Publishing)

Willes, A. J. 2002, JGR, 107, 1061

Wilson, R., Calway, A. D., \& Pearson, E. R. S. 1992, IEEE Trans. Inform. Theory, 38(2), 674

Zaitsev, V. V., Zlotnik, E. Ya., \& Shaposhnikov, V. E. 1986, A\&A, 169,345 